\title{
Spatial resolution limits for synchrotron-based infrared spectromicroscopy
}

\author{
Erika Levenson ${ }^{\mathrm{a}}$, Philippe Lerch ${ }^{\mathrm{b}}$, and Michael C. Martin ${ }^{\mathrm{a}}$ \\ ${ }^{a}$ Advanced Light Source Division, Lawrence Berkeley National Laboratory, 1 Cyclotron Road, Berkeley, CA 94720, USA \\ ${ }^{b}$ Swiss Light Source, Paul Scherrer Institut, 5232 Villigen, Switzerland
}

\begin{abstract}
Detailed spatial resolution tests were performed on beamline 1.4.4 at the Advanced Light Source synchrotron facility in Berkeley, CA. The high-brightness synchrotron source is coupled at this beamline to a Thermo-Electron Continu $\mu \mathrm{m}$ XL infrared microscope. Two types of resolution tests in both the mid-IR (using a KBr beamsplitter and an MCT-A* detector) and in the near-IR (using a $\mathrm{CaF}_{2}$ beamsplitter and an InGaAS detector) were performed and compared to a simple diffractionlimited spot size model. At the shorter wavelengths in the near-IR the experimental results begin to deviate from only diffraction-limited. The entire data set is fit using a combined diffraction-limit and demagnified electron-beam source size model. This description experimentally verifies how the physical electron beam size of the synchrotron source demagnified to the sample stage on the endstation begins to dominate the focussed spot size and therefore spatial resolution at higher energies. We discuss how different facilities, beamlines, and microscopes will affect the acheivable spatial resolution. (C) 2008 Elsevier Science. All rights reserved
\end{abstract}

Keywords: Synchrotron; Spatial Resolution; Microscopy; FTIR; Spectromicroscopy; Imaging; Diffraction; Emittance

\section{Introduction}

Synchrotron infrared (IR) beamlines provide diffraction-limited spatial resolution for spectromicroscopy with $100-1000$ times higher brightness than a conventional thermal globar source [1-4] enabling a wide variety of new science at small spatial scales $[3,4]$. We have previously experimentally verified the diffraction-limited performance through the mid-IR at the Advanced Light Source (ALS) [5]. However as the synchrotron emission wavelength becomes shorter, the physical size of the electron beam will dominate the focused photon beam size.
The source size of a synchrotron light beam can be approximated well by adding in quadrature the effects of diffraction, the electron beam size, and the projected size of the emitting region $[1,6]$. This beam is then imaged onto a sample via beamline optics that have an overall demagnification factor, $\mathrm{m}$. The spot size can therefore be written as

$$
m \sqrt{(d \lambda)^{2}+\sigma_{t}^{2}}
$$

where $\mathrm{d}$ is a diffraction limit factor (depends on which resolution model is chosen, as described later), $\lambda$ is the wavelength of light, and $\sigma_{\mathrm{t}}$ is the transverse synchrotron electron beam size. The transverse electron beam size is given by

$$
\sigma_{t}=\sqrt{\beta_{t} \varepsilon_{t}+\eta_{t}^{2}\left(\frac{\sigma_{E}}{E}\right)^{2}}
$$

where $\beta_{t}$ is the beta-function, $\varepsilon_{t}$ is the emittance, $\eta_{t}$ is

\footnotetext{
* Corresponding author. Tel.: +1-510-495-2231; fax: +1-510-495-2067; e-mail: MCMartin@lbl.gov.
} 
the dispersion (all in the transverse direction; $t$ can be $\mathrm{x}$ or $\mathrm{y})$, and $\sigma \mathrm{E} / \mathrm{E}$ is the energy spread [7]. These parameters are specific to each synchrotron light sournce and the specific beamline photon port.

\section{Experiment}

We performed lateral resolution experiments as a function of wavelength in the mid- and near-IR using a Thermo-Electron Continu $\mu \mathrm{m}$ XL microscope and Nexus 870 FTIR bench at ALS beamline 1.4.4. The beamline collects $10 \mathrm{mrad}$ vertical by $40 \mathrm{mrad}$ horizontal collection from the bending magnet source. A pair of cylindrical mirrors are used to collimate this source before the light is steered into the emission port of the FTIR bench. The IR microscope focuses the beam onto the sample using all reflective $15 \times$ or $32 \times$ cassegrain objectives with numerical apertures of 0.58 and 0.65 respectively. The results presented here were obtained with the $32 \times$ objective. The sample stage is an automated Prior Scientific H101 stage with step sizes as small as 0.1 microns. All measurements were done in reflection mode without any apertures in the light path, and the results presented here detail the $y$ direction cross-sections of the focused spot.

We previously published mid-IR resolution test results showing the resolution is indeed diffractionlimited [5], so for this study we concentrate on the near-IR. An MCT-A* detector was used for 2000 $\mathrm{cm}^{-1}$ to $7000 \mathrm{~cm}^{-1}$, and an InGaAs detector was utilized for $5000 \mathrm{~cm}^{-1}$ to $11000 \mathrm{~cm}^{-1}$. A CaF beamsplitter was used to cover this entire range.

Spatial resolution tests were performed using a high-resolution USAF 1951 3-Bar Resolving Test Chart [8] from Applied Image Inc. (Rochester, NY). It has a chrome metal coating on a glass substrate with the resolution test structures patterned in a negative image up to a spatial frequency of 512 cycles/mm (smallest is Group 9, Element 3).

Two types of resolution tests were done as defined in more detail in Ref. [5]: a step edge (or knife edge) test, and a Rayleigh's criterion test. These two definitions are well known from the literature, however it is important to note that they yield different numerical results since they are based on different definitions. The Rayleigh imaging criterion will give a higher resolution than the FWHM stepedge analysis by a factor 0.617 .

\subsection{Step Edge Resolution Test Results}

Profiles were measured for each analyzed wavelength, then the derivative of each line profile was fit to a Gaussian. Fig. 1 shows the FWHM resolution versus wavelength for both detectors. A simple linear fit to the data yields a resolution for the synchrotron source of $(0.73 \pm 0.04) \lambda$.

This, however, underestimates the slope of the real data, and the data deviate from the fit at shorter wavelengths. An improved and more physically meaningful fit is obtained by using equation 1 . In this case the best fit results in the diffraction portion of the data is given by $(0.81 \pm 0.02) \lambda$, with a demagnified electron beam size of $0.71 \pm 0.11 \mu \mathrm{m}$.

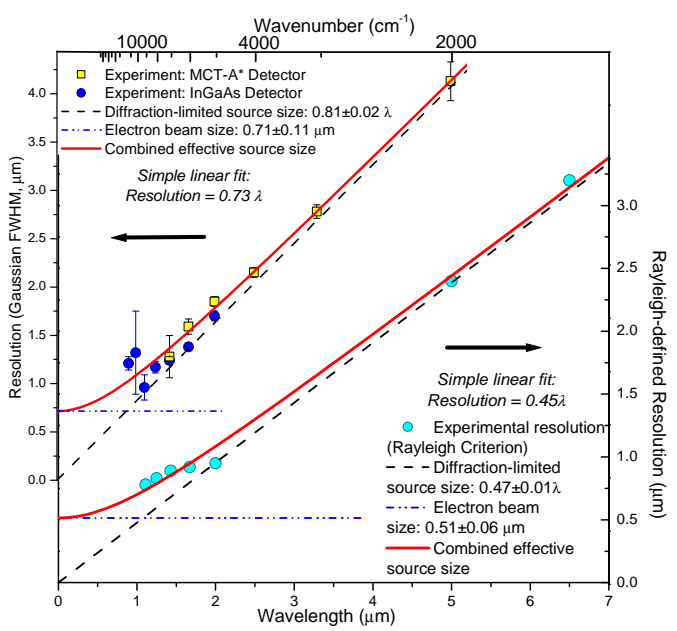

Fig 1. Step edge and Rayleigh resolution results as a function of wavelength. Fits to the data to the effective source size discussed in the text is shown in red, with the diffraction and electron beam source size components of each fit shown with dashed lines.

\subsection{Imaging Resolution Test Results}

Imaging tests were done using the USAF chart and Rayleigh's criterion for $\lambda=6.5-1.11 \mu \mathrm{m}$. The measured resolutions of all wavelengths analyzed are 
presented in Fig. 1. A simple linear fit to the data yields a resolution of $(0.45 \pm 0.02) \lambda$.

Again, however, this simple linear fit does not adequately follow the data point particularly at shorter wavelengths, nor does it intercept the origin. The experimental imaging data clearly show deviation from a simple diffraction-limited spot size at short wavelengths. An improved fit is found using equation 1 with the diffraction portion of the data given by $(0.47 \pm 0.01) \lambda$, and a demagnified electron beam size of $0.51 \pm 0.06 \mu \mathrm{m}$.

\section{Analysis}

As noted above, the step-edge and imaging tests use different resolution definitions differing by a factor of 0.617 . Indeed the diffraction portion of the best fits to Eqn. 1 are different by a factor of $0.59 \pm$ 0.03 and the electron beam size portions of the fits are different by a factor of $0.72 \pm 0.20$, both within the error bars of the ideal factor. We conclude that the two resolution tests give consistent results.

The electron beam source size for the 1.4 (22.6 degree) bending magnet port at the ALS is $\sigma_{\mathrm{x}}=65$ $\mu \mathrm{m}$ and $\sigma_{\mathrm{y}}=52 \mu \mathrm{m}[10]$. These are one sigma values, and the FWHM beam size is 2.35 times larger. Thus, the electron beam size is $153 \mu \mathrm{m}$ in the horizontal and $122 \mu \mathrm{m}$ in the vertical dimension.

The magnification factor $\mathrm{m}$ for the 1.4 beamline is given by the ratio of the focal distance of the collimating mirror to the focal distance of the microscope objective focusing the light onto the sample. The beamline 1.4.4 optics image the vertical synchrotron emission to the y-direction on the sample stage which is the direction in which the spot size measurements are detailed above. The magnification factor for the $32 \times$ objective is $\mathrm{m}=171$, and thus we would predict that the FWHM electron beam size of $122 \mu \mathrm{m}$ is imaged onto the sample stage to $0.71 \mu \mathrm{m}$, FWHM. Or using the Rayleigh criterion, the imaged beam size is $0.44 \mu \mathrm{m}$. These values are in good agreement with experimental data of the demagnified electron beam source size fits of $0.71 \pm 0.11 \mu \mathrm{m}$, and $0.51 \pm 0.06 \mu \mathrm{m}$, for the two resolution definitions, respectively. For the $32 \times$ objective with $\mathrm{NA}=0.65$, we expect the diffraction limited performance of this objective to be $0.47 \lambda$. This is in excellent agreement with the fit to our measured data of $(0.47 \pm 0.01) \lambda$.

\subsection{Resolution of different synchrotron beamlines}

The achievable transverse resolution of a given synchrotron beamline is a combination of the beamline optics (demagnification factor), the electron beam source size which is dependent on the specific machine and photon port parameters, and the final focusing objective in the IR microscope. ThermoElectron's $15 \times$ and $32 \times$ objectives have relatively large N.A.'s, whereas Bruker Optics' $15 \times$ and $36 \times$ objectives have longer working distances but smaller N.A.'s of 0.4 and 0.5 , respectively. Using such objectives, the effects of the electron beam source

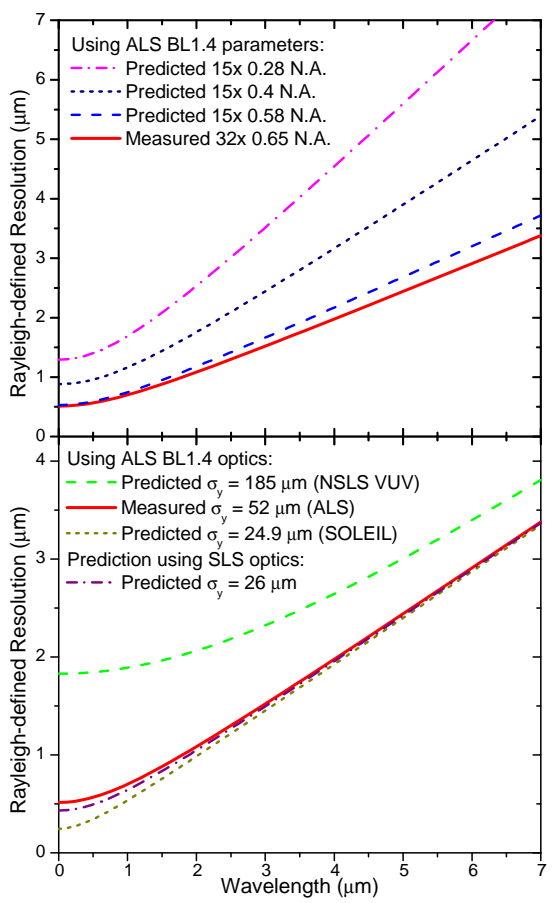

size will start at longer wavelengths and will limit the

Fig 2. Predicted resolutions for different numerical aperture objectives, and different electron beam source sizes at selected synchrotron light sources.

ultimate lateral spatial resolution achievable. If we were to use the Bruker $15 \times$ objective $(\mathrm{N} . \mathrm{A}=0.4)$ on the ALS IR beamline with all other optics being the same, we should obtain a spot size given by $0.76 \lambda$ 
for the diffraction-limit, and $0.88 \mu \mathrm{m}$ imaged electron beam size. Examples of the predicted resolution as a function of wavelength using different N.A. objectives are plotted in the upper panel of Fig. 2 .

If the ALS IR beamline was placed on the smallest source size port at the ALS the vertical electron beam size would be $\sigma_{\mathrm{y}}=15 \mu \mathrm{m}$, or a factor of 3.5 smaller. This would have minimal effect to the resolution in most of the mid-IR, but the diffraction-limited performance would extend further into the near-IR and a significant improvement in resolution could be achieved for wavelengths shorter than about 2 microns. If the same beamline was built at the NSLS VUV ring (a 2nd generation synchrotron which has IR microscopy beamlines with similar IR microscopes), the vertical electron beam source size is $\sigma_{\mathrm{y}}=185 \mu \mathrm{m}$ [11], or about 3.5 times larger than the ALS 1.4 port. The electron beam source size will play a more dominant role in the total effective resolution, yielding about 0.5 microns larger spot size at $\lambda=5 \mu \mathrm{m}\left(2000 \mathrm{~cm}^{-1}\right)$, extending to 1 micron larger spot size at $\lambda=2 \mu \mathrm{m}\left(5000 \mathrm{~cm}^{-1}\right)$ compared to ALS BL1.4. An IR microscopy beamline is being built at the new SOLEIL synchrotron (France) with a planned $\sigma_{\mathrm{y}}=24.9 \mu \mathrm{m}$ [12], so assuming similar magnification optics to the ALS the diffraction limited performance will continue to wavelengths shorter than 1 micron $\left(10,000 \mathrm{~cm}^{-1}\right)$.

The source size at the IR port currently being commissioned at the Swiss Light Source (SLS) storage ring is expected to be $\sigma_{\mathrm{x}}=52 \mu \mathrm{m}$ and $\sigma_{\mathrm{y}}=26$ $\mu \mathrm{m}$. The magnification factor $\mathrm{m}$ at the SLS IR beamline is 86 (with a 0.58 N.A objective) or 82 (with a 0.61 N.A objective). This means that the $\sigma_{\mathrm{y}}=$ $26 \mu \mathrm{m}$ vertical electron beam size will be imaged to the sample with a FWHM of $0.7 \mu \mathrm{m}$, or $0.43 \mu \mathrm{m}$ using the Rayleigh definition. These values are very close to those measured at the ALS so we would predict very similar resolution capabilities assuming the use of a similar microscope objective. The predicted resolution from several synchrotron source sizes are shown in the lower panel of Fig. 2.

The under development NSLS-II storage ring [13] is planned to perform at close to the theoretical minimum emittance [7] possible for a storage ring. The source size in the bending magnet ports will be $\sigma_{\mathrm{x}}=44.2 \mu \mathrm{m}$ and $\sigma_{\mathrm{y}}=15.7 \mu \mathrm{m}$. This is almost a factor of two smaller vertical beam size than
SOLEIL, and so the diffraction limited performance should extend to approximately $\lambda=400 \mathrm{~nm}$, in the visible. Different focusing optics could extend this range even further towards the VUV.

\section{Acknowledgements}

We would like to thank Fernando Sannibale. This work and the Advanced Light Source are supported by the Director, Office of Science, Office of Basic Energy Sciences, Materials Sciences Division, of the U.S. Department of Energy under Contract No. DEAC02-05CH11231 at Lawrence Berkeley National Laboratory.

\section{References}

[1] J. A. Reffner, P. A. Martoglio, and G. P. Williams, Rev. Sci. Instrum. 66, 1298 (1995); G. L. Carr, J. A. Reffner, and G. P. Williams, Rev. Sci. Instrum. 66, 1490 (1995) ; G. L. Carr, Rev. Sci. Instrum. 72, 1613 (2001).

[2] Michael C. Martin and Wayne R. McKinney, Proceed. Mater. Res. Soc., 524, 11 (1998); Michael C. Martin and Wayne R. McKinney, Ferroelectrics, 249(1-2), 1-10, (2001).

[3] Hoi-Ying N. Holman, Michael C. Martin, and Wayne R. McKinney, Spectroscopy - An International Journal 17(2-3), 139-159 (2003); Hoi-Ying N. Holman and Michael C. Martin, Advances in Agronomy 90, 79-127 (2006).

[4] Paul Dumas and Mark J. Tobin, Spectroscopy Europe 15 (6), 17-23 (2003).

[5] Erika Levenson, Philippe Lerch, and Michael C. Martin, Infrared Physics and Technology 49, 45-52 (2006).

[6] C.J. Hirschmugl, Ph.D. Thesis, Yale University (1994).

[7] K.-J. Kim, "Characteristics of Synchrotron Radiation," in Physics of Particle Accelerators, AIP Conf. Proc. 184 (Am. Inst. Phys., New York, 1989).

[8] MIL-STD-150A, Section 5.1.1.7, USAF 1951 3-Bar Resolving Power Target; also see http://www.efg2.com/Lab/ImageProcessing/TestTargets/

[9] John C. Russ, The Image Processing Handbook, CRC Press, 2002, p. 320.

[10] http://www.als.lbl.gov/als/techspecs/srparameters.html

[11] http://www.nsls.bnl.gov/facility/accelerator/vuv/ vuv parameters.pdf

[12] http://www.synchrotron-soleil.fr/portal/page/portal/ SourceAccelerateur/ParametresPointsSources

[13] http://www.bnl.gov/nsls2/project/source_properties 